\title{
7. Deutscher Strafverteidigertag
}

Seinem Ursprung nach stellt sich der seit dem Jahre 1977 alljährlich durchgeführte Deutsche Strafverteidigertag als Kind der Not dar. Im Zusammenhang mit den großen Staatsschutzprozessen der siebziger Jahre in Stuttgart-Stammheim und anderswo war die Strafverteidigung ins öffentliche Gerede und Visier geraten. Ganze Gruppen von Verteidigern wurden verdächtigt und bezichtigt, ihre Stellung als Rechtsanwälte dazu zu mißbrauchen, das allgegenwärtig-terroristische Geschäft ihrer Mandanten selbst zu betreiben oder zu fördern. "Der Verteidiger als Staatsfeind « erwies sich als publikumswirksames Stück und wurde über Verteidigerausschlüsse und mittels aufwendig durchgeführter Straf- und Ehrengerichtsverfahren gegen die angeblich so bösen Vertreter der Zunft, die da zu Werke waren, in Szene gesetzt. In der Zeit von 1974 bis 1977 griff der Gesetzgeber - seinerzeit noch der sozialliberale mit starker Hand nach der Strafprozeßordnung und nahm die Verteidigung an die kurze Kandare.

Die Strafverteidiger, die sich von dieser Entwicklung aktuell oder potentiell bedroht sahen, artikulierten ihren Protest gegen das trübe Schicksal, welches die Staatsgewalt den strafprozessualen Verteidigungsrechten da nach Kräften zu bereiten trachtete, zunächst auf mehr oder minder informellen Zusammenkünften. Ab Mitte der siebziger Jahre etwa bildeten sich dann - dem Vorbild der seinerzeit bereits existierenden Vereinigung Berliner Strafverteidiger e. V. folgend - zunächst in Hamburg und Niedersachsen und in der Folgezeit dann auch in Hessen und in Bayern regionale, jeweils als Vereine eingetragene Strafverteidigervereinigungen. Diese erfreuten sich alsbald eines Zulaufes, der weit über den Kreis ihrer Initiatoren hinausging. Ursache hierfür dürfte zum einen gewesen sein, daß die Berufsgruppe der Strafverteidiger innerhalb der klassischen Organisationen der Anwaltschaft, das heißt vor allem innerhalb des Deutschen Anwaltsvereines, von jeher eher ein Schattendasein führte. Der Zulauf, den die Strafverteidigervereinigungen im Anschluß an ihre Gründung fanden, dürfte sich zum anderen aber auch daraus erklären, daß Strafverteidigung bei denjenigen Juristen, die sich Ende der sechziger, Anfang der siebziger Jahre für den Beruf des Rechtsanwaltes entschieden, auf größeres Interesse und auf größeren beruflichen Zuspruch als bei den vorangegangenen Generationen gestoßen ist.

Die genannten Strafverteidigervereinigungen sind Veranstalter und Träger des seit dem Jahre 1977 alljährlich durchgeführten Deutschen Strafverteidigertages.

Die Thematik der Strafverteidigertage stand von Anfang an im Zeichen der Defensive gegen den repressiven Geist, der seit Mitte der siebziger Jahre den Umgang des Gesetzgebers mit der Strafprozeßordnung kennzeichnet. Allerdings machte gerade der in der Zeit vom I 5 . bis 17. 4. 1983 in Frankfurt am Main durchgeführte 7. Deutsche Strafverteidigertag seinem Ablauf wie seinem äußeren Rahmen nach deutlich, $\mathrm{da} ß$ der Strafverteidigertag in seiner noch jungen Geschichte bereits erhebliche Veränderungen durchlaufen hat. In seinen Anfängen schien der Strafverteidigertag im Verhältnis zu den traditionellen Großveranstaltungen der praktischen Jurisprudenz der Bundesrepublik - etwa dem Deutschen Juristentag oder dem Deutschen Richtertag - das etablierte juristische Spektrum noch eher vom gleichsam alternativen Rande her zu bewegen und zu beleben. Inzwischen hat er sich jedoch - der 7 . Strafverteidigertag hat dies nachdrücklich bewiesen - zu einer Art nationaler Institution gemausert. Sein äußerer Rahmen ist nicht mehr durch die Kargheit der Dissidenz, sondern durch weitläufige Gediegenheit geprägt. Die einleitenden Worte zur Begrüßung atmen manches »möge, möge ...«, der Vertreter des leider persönlich verhinderten Hessischen Ministers der Justiz entbietet vom blumengeschmückten 
Rednerpult eine artige Grußbotschaft, Richter - unter ihnen ein Vorsitzender Richter am Bundesgerichtshof -, Professoren und Ministerialräte in Bundesjustizministerium werden als Diskussionsteilnehmer und Debattenredner geworben und gewonnen. Wo früher der trotzig-republikanische Gestus der harschen Kritik an einer legislativen und judikativen Obrigkeit, deren Staatsverständnis manch vorkonstitutionelle Trübung aufwies, den Diskussionen nicht fremd war, dominiert heute eher der fachjuristische Diskurs.

Noch in der zweiten Hälfte der siebziger Jahre standen gerade diejenigen Strafverteidiger, deren Organisationen den Strafverteidigertag aus der Taufe gehoben haben, bei der etablierten Jurisprudenz der Bundesrepublik, auch und gerade bei der Richterschaft und ihren Organisationen, im Ruch der aufmüpfigen Widersetzlichkeit und Radikalität. Ausgrenzung, Mißtrauen und Verdacht waren lange Zeit die Folge. Auf Seiten der durch die Entwicklung in den siebziger Jahren arg gebeutelten und gerupften Verteidiger zeitigte die Erbitterung über die Ächtung, die an ihnen versucht wurde, umgekehrt gelegentliche Neigungen zum politischen Sektierertum. Der Verlauf des 7. Strafverteidigertages hat deutlich gemacht, daß inzwischen im gegenseitigen Umgang miteinander, auch und gerade im Verhältnis von Strafverteidigern und Richterschaft, die Tendenz der integrativen Annäherung vorherrscht. Die vormals mit so viel Mißtrauen wahrgenommenen Verteidiger scheinen im sozialen Gefüge namentlich der Dritten Gewalt an Prestige und Einfluß gewonnen zu haben. Zu fragen ist allerdings, ob dies auch zu einer Stärkung der rechtspolitischen Durchschlagskraft der zentralen und traditionellen, am Schutz der strafprozessualen Verteidigungsrechte orientierten Anliegen des Strafverteidigertages geführt hat.

Schwerpunktthema des diesjährigen Strafverteidigertages waren neuere Initiativen, die darauf abzielen, einmal mehr dem ohnedies bereits eng gewordenen Spielraum der Verteidigung im Strafverfahren zu Leibe zu rücken. Zum einen geht es um "Vorschläge zu gesetzlichen Maßnahmen zur Entlastung der Gerichte und Staatsanwaltschaften in der Strafgerichtsbarkeit -, Stand: I. 3. 1982", präsentiert von der Konferenz der Justizminister und -senatoren des Bundes und der Länder (JuMiKo), ein CEuvre, das bei kritischen Eingeweihten zu Recht als sogenannte "Horrorliste diskutiert wird; zum anderen geht es um eine noch aus den letzten Tagen der sozialliberalen Koalition, das heißt noch aus der Amtszeit des früheren Bundesjustizministers Schmude und seinem Ministerium stammenden "Referentenentwurf(es) eines Gesetzes zur Änderung strafverfahrensrechtlicher Vorschriften (Strafverfahrensänderungsgesetz 1983 - StVÄG)) (RefE). Dieser verhält sich gegenüber dem, was die JuMiKo empfiehlt, zwar noch relativ moderat, bietet zum Recht der Pflichtverteidigung sogar manch über die bisherige Regelung hinausgehendes liberales Bonbon an (z. B. Beiordnung eines Pflichtverteidigers bereits nach einem Monat Untersuchungshaft), bezeugt jedoch in anderen Bereichen, z. B. zum Recht der Richterablehnung oder zur Frage eines verkürzten Instanzenweges im Verfahren vor dem Amtsgericht ebenfalls eine ungute Neigung zum »kurzen Prozeß«. Angesichts der seit dem 6. 3. 1983 im Deutschen Bundestag bestehenden Mehrheitsverhältnisse dürfte die Horrorliste der JuMiKo gegenüber dem Referentenentwurf des Bundesjustizministers im übrigen ohnedies die besseren legislatorischen Erfolgsaussichten haben - eine bedrückende Perspektive, wenn man sich die Kernstücke vor Augen führt, die da zum neuen Strafverfahrensrecht werden sollen.

- Im Verfahren vor dem Amtsgericht wird das förmliche Beweisantragsrecht des $\$ 244$ Abs. 3 bis s StPO abgeschafft; über eine vom Angeklagten oder seiner Verteidigung begehrte Beweiserhebung entscheidet das Gericht nach seinem freien Ermessen. Die Möglichkeit einer Sprungrevision gegen amtsgerichtliche Urteile entfällt (dies sieht auch der Referentenentwurf vor). Gegen Berufungsurteile soll die Revi- 
sion nur noch zur Wahrung der Rechtseinheit oder zur Fortbildung des Rechtes, also nur noch auf besonderen Zulassungsbeschluß hin stattfinden (auch hier hat sich der Referentenentwurf angeschlossen).

Fazit: Dem Angeklagten sind gnädige Richter zu wünschen.

- So unersprießliche Dinge wie die Erörterung der möglichen Befangenheit eines Richters sollen nach Möglichkeit das Licht der Hauptverhandlung meiden. Ablehnungsgründe, die aus der Zeit vor Beginn der Hauptverhandlung datieren, sind (ebenso dem Referentenentwurf zufolge) "unverzüglich nach Mitteilung der Besetzung des Gerichtes oder, falls eine solche Mitteilung unterblieben ist, nach Beginn der Hauptverhandlung geltend zu machen.*

Motto: Der Herr und Gebieter der Hauptverhandlung als strahlende Lichtgestalt, nicht nur unbefleckt, sondern nach Möglichkeit auch unbefleckbar.

- Das Recht, Anträge zu begründen, Fragen zu stellen und Erklärungen im Sinne des $₫ 247$ StPO abgeben zu können, soll »einem Prozeßbeteiligten « (dreimal dürfen wir raten, welcher Beteiligte hier wohl gemeint ist) vom Vorsitzenden entzogen werden können, wenn es nach seinem Dafürhalten an einem "sachlichen Zusammenhang zum Verfahren « fehlt oder eine "sachlich nicht gebotene Verzögerung der Hauptverhandlung « Folge der Rechtswahrnehmung ist.

Im Klartext: Die Entscheidung darüber, was für die Verteidigung eines Angeklagten sachdienlich und geboten ist, wird der alleinigen Disposition des Angeklagten und seines Verteidigers entzogen und in letzter Instanz der Ermessensentscheidung des Vorsitzenden unterstellt. Bleibt nur zu hoffen, daß dieser von der Sache, um die es dem Angeklagten und seiner Verteidigung geht, auch etwas versteht.

Der kurze Prozeß, um den sich die Vorschläge der JuMiKo und in ihrem moderateren Gefolge der Referentenentwurf des Bundesjustizministeriums bemühen, soll der Generaltendenz der vorliegenden Änderungsempfehlungen zufolge einmal mehr über die (weitere) Reduzierung der zum Schutze des Beschuldigten oder Angeklagten geschaffenen Abwehrrechte erreicht werden. Da sollen - unter Verwässerung der Konzentrationsmaximen - die bisher möglichen Unterbrechungsfristen für die Hauptverhandlung ebenso verlängert werden wie die Fristen für die vom Oberlandesgericht vorzunehmende Haftprüfung; auf die Verlesung von Urkunden in der Hauptverhandlung soll fürderhin auch ohne Zustimmung der Verteidigung verzichtet werden können (wenn Richter und Schöffen die betreffenden Urkunden gelesen haben und die übrigen Verfahrensbeteiligten hierzu Gelegenheit hatten), den Vorschlägen der JuMiKo zufolge soll dies sogar - Unmittelbarkeitsmaxime hin oder her - für die Verlesung von Protokollen in den Fällen der $\$ \$ 253,254$ StPO (frühere Aussagen von Zeugen oder Angeklagten) gelten.

Interessant und aufschlußreich ist, was die Autoren der strafprozessualen Änderungsw ünsche, die auf dem 7. Strafverteidigertag in Frankfurt am Main diskutiert wurden, zu deren Begründung vorzutragen wissen. Während der Gesetzgeber der siebziger Jahre zur Begründung der von ihm behaupteten Notwendigkeit, Verteidigungsrechte einschränken zu müssen, noch stereotyp auf angeblich "rechtsmißbräuchlich« agierende Verteidiger verwies, beschwört der Gesetzgeber der achtziger Jahre in erster Linie das Bild einer Strafjustiz, die alsbald in einer gurgelnden Flut von Verfahren zu ertrinken droht, wenn ihr nicht durchgreifende legislative Entlastung zuteil wird. Die Mär vom allenthalben rechtsmißbräuchlich zu Werke gehenden Verteidiger findet sich allerdings bei näherem Hinsehen auch noch in mancher Begründung der jetzt neu vorliegenden Änderungsvorschläge wieder. Dies gilt für die in den Vorschlägen der JuMiKo vorgesehene Einschränkung des Antragsbegründungs-, des Frage- und des Erklärungsrechtes ebenso wie für die Begründung des Referentenentwurfes des Bundesjustizministeriums zur Frage der Vorverlegung des 
Zeitpunktes von Richterablehnungen. Da scheint sich noch so manches Mütchen, das sich in den "Reformen " der siebziger Jahre vermutlich zu kurz gekommen sah, an und in den neuen Entwürfen gekühlt zu haben.

Im Hintergrund der zu den Stichworten Prozeßflut und Justizüberlastung vorgebrachten Begründungen der neuen Änderungsentwürfe steht die seit einiger Zeit von hohen und höchsten Repräsentanten der Justiz der Bundesrepublik propagierte These von der »knappen Ressource Recht«. So lautete die Überschrift eines in der ZRP 198I/I I I ff. veröffentlichten Beitrages des Präsidenten des Bundesgerichtshofes, Professor Pfeiffer. ${ }^{1}$ Zum Thema einer Justiz, deren baldiger Exitus qua Überlastung zu befürchten ist, haben sich inzwischen auch der Präsident und der Vizepräsident des Bundesverfassungsgerichtes, letzterer in einem Vortrag auf dem diesjährigen Deutschen Richtertag in München, öffentlich zu Wort gemeidet.

Die Formulierung von der »knappen Ressource Recht« legt die Vorstellung nahe, das Recht drohe aufgrund seiner sozialen Förderkosten zu einem zur Neige gehenden Rohstoff zu werden, dessen weitere Verfügbarkeit allenfalls bei sparsamem Verbrauch gewährleistet ist. $\mathrm{Zu}$ Recht wies Professor Ulrich Preuß in einem Vortrag zum 7. Strafverteidigertag mit dem Thema "Strafverfahrensrecht und politische Kultur « darauf hin, daß die Propagierung oder Förderung solcher Vorstellungen sehr wohl mit der Gefahr einer Korrumpierung öffentlichen Bewußtseins verbunden ist. Die Vorstellung einer Justiz, die ihre Dienste gleichsam nur noch auf karg bemessene und verteilte Rechtsmittelkarten hin zu erbringen bereit oder in der Lage ist, vermag rechtsstaatlichen Postulaten nicht standzuhalten. Auch der vielbeschworenen Wertsetzung des Grundgesetzes dürfte der Gedanke eines Rechts als bloßer Funktion seiner sozialen Kosten einigermaßen fremd sein.

Die Horrorliste der JuMiKo und die Vorschläge des Referentenentwurfes des Bundesjustizministeriums verfielen auf dem 7. Deutschen Strafverteidigertag dem Verdikt einhelliger Ablehnung durch die versammelten Strafverteidiger. Dabei verlief die in der Arbeitsgruppe I geführte Diskussion der Änderungsvorschläge auffallend emotionsfrei und leidenschaftslos. Die vielen an der Diskussion beteiligten Verteidiger bemühten sich redlich, wenn auch im Ergebnis ohne Erfolg, dem Inhalt der debattierten Änderungsempfehlungen die Vorgabe rechtsstaatlicher Rationalität zuteil werden zu lassen. Professor Peter Rieß, der, was die legislative Einschränkung von Verteidigungsrechten angeht, als langjähriger Ministerialrat im Bundesjustizministerium sicherlich über mehr Täter- als Opfererfahrung verfügen dürfte, hatte in den Diskussionen der Arbeitsgruppe I deshalb gut reden. Jedoch legte auch er Wert auf kritische Distanz zumindest zu den verteidigungsfeindlichsten Vorschlägen der JuMiKo.

Daß sich der Deutsche Strafverteidigertag überhaupt und einmal mehr mit strafprozessualen Änderungsbestrebungen der Art, wie sie die Vorschläge der JuMiKo oder der Referentenentwurf des Bundesjustizministeriums vorsehen, auseinandersetzen mußte, statt gegenüber dem in der Strafprozeßordnung derzeit bestehenden legislativen Zustand eigene Vorstellungen über die Gestaltung einer freien Verteidigung offensiv vortragen zu können, ist allerdings alleine schon ein deutlicher Hinweis darauf, wie schwach und nach wie vor gefährdet es in der Bundesrepublik um das Rechtsinstitut der Verteidigung bestellt ist.

Deutlich wurde auf dem 7. Strafverteidigertag im übrigen auch, daß die Ablehnung der neuen legislativen Vorhaben, die Verteidigungsrechte weiter einzuschränken,

1 Zur Ehre des Präsidenten des Bundesgerichtshofs sei darauf hingewiesen, daß dieser sich inzwischen in einem vom "Spiegel" in der Ausgabe vom 18.7. 1983 veröffentlichten Interview in überaus kritischer Weise über die Vorschläge zur Änderung der Strafprozeßordnung geäußert hat. 
inzwischen quer durch alle Anwaltsorganisationen der Bundesrepublik zu verlaufen scheint. Noch in den siebziger Jahren genügte der Hinweis auf die angeblich so schwarzen Schafe der Zunft, um den Deutschen Anwaltsverein etwa dazu zu veranlassen, einem großen Teil der vom Gesetzgeber zu dieser Zeit beschlossenen Einschränkungsgesetze seine Zustimmung nicht zu versagen. Diese Zeiten sind vorbei. So gehörte zu den schärfsten Kritikern der neuen Änderungsvorschläge in Frankfurt am Main Dr. Hans Dahs, Experte für Straf- und Strafverfahrensrecht im Deutschen Anwaltsverein und Mitautor des klassischen "Handbuches des Strafverteidigers«.

An den Diskussionen und Debatten des 7. Strafverteidigertages in Frankfurt am Main waren auf entsprechende Einladung hin Richter in erklecklicher Zahl beteiligt. Die Diskussionen zwischen Richtern und Strafverteidigern waren sicherlich dazu geeignet, das eine oder andere berufsrollenbedingte wechselseitige Vorurteil im Verhältnis miteinander zu beseitigen. Im Rahmen einer zum Abschluß des 7. Strafverteidigertages veranstalteten Podiumsdiskussion (Thema: "Welche Strafjustiz will dieses Land sich leisten? «) ging Professor Detlef Krauss von der juristischen Fakultät der Universität Basel, der in Frankfurt am Main engagiert für die Belange der Verteidigung stritt, sogar so weit, ein rechtspolitisches Bündnis von Strafverteidigern und Richterschaft zum Schutze der Freiheit der Verteidigung zu fordern. Ob eine solche Forderung allerdings realistisch ist, muß füglich bezweifelt werden. Dagegen spricht zunächst die Erfahrung, daß die mit dem Strafrecht befaßten Teile der Richterschaft, die am oberen Ende der Hierarchie der Dritten Gewalt ihres Amtes walten, in ihrer überwiegenden Mehrheit einem Staats- und Rechtsverständnis zu huldigen scheinen, das den Belangen einer freien Verteidigung - vorsichtig ausgedrückt - keine besondere rechtspolitische Priorität beimißt. (So manches, was über die dem Straf- und Staatsschutzrecht gewidmeten Diskussionen in der Trierer Richterakademie gemunkelt und geflüstert wird, würde auch weniger vorsichtige Formulierungen rechtfertigen.) Zum anderen erwecken auch die öffentlichen Verlautbarungen des Deutschen Richterbundes nicht gerade den Eindruck, der unabdingbare Schutz der freien Verteidigung könnte dieser Berufsorganisation eine mit Vorrang versehene Herzensangelegenheit sein.

Allerdings gibt es auch innerhalb der Richterschaft der Bundesrepublik (ebenso wie bei den Staatsanwälten) organisationsähnliche Gruppierungen, deren republikanischer und demokratischer Selbstanspruch die korporativen Elemente des traditionellen Selbstverständnisses deutscher Richter in erfreulicher Weise verdrängt hat. $\mathrm{Zu}$ erwähnen ist hier vor allem der sogenannte Richterratschlag, eine Gruppe von Richtern und Staatsanwälten, die sich innerhalb der Gewerkschaft ÖTV zusammengefunden hat. Es mag durchaus sein, daß die Mehrzahl ihrer Mitglieder nicht an den oberen Gerichten (oder Anklagebehörden) des Bundes oder der Länder tätig sind. Für den Strafverteidigertag, der sich als Institution um den Schutz der Verteidigungsrechte von Beschuldigten und Angeklagten und damit um den Schutz und den Bestand von Bürgerrechten überhaupt bemüht, dürften Gruppen wie der Richterratschlag dennoch oder vielleicht gerade deswegen glaubwürdige und wirksame rechtspolitische Bündnispartner sein. Als Referenten oder Podiumsredner waren Vertreter solcher Gruppen zum 7. Strafverteidigertag jedoch nicht geladen - ein Versäumnis, das in Zukunft nicht wiederholt werden sollte.

Rupert v. Plottnitz 